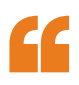

ESCRT-III

components ...

as well as

VPS4A were

only transiently

recruited to the

HIV- 1 and EIAV

particles at a late

stage

\title{
ESCRTing retroviruses to the exit
}

The endosomal sorting complex required for transport (ESCRT) machinery is required for several membrane scission events in eukaryotic cells, including those involved in the budding of some enveloped viruses from the plasma membrane. In mammals, the ESCRT machinery consists of three distinct complexes (ESCRT-I, ESCRT-II and ESCRT-III) that together contain more than 20 proteins and additional associated factors, such as the vacuolar protein sorting-associated protein $4 \mathrm{~A}$ (VPS4A) ATPase. ESCRT components are thought to be recruited to the site of viral budding in an ordered manner, with ESCRT-I proteins acting early in the pathway and ESCRT-III proteins acting late in the pathway, although a detailed understanding of the process has proved to be elusive. Three studies now shed light not only on the kinetics of the recruitment of ESCRT proteins to the site of viral budding but also on the composition of the complexes involved.

One of the major obstacles to studying the dynamics of ESCRT components has been the adverse

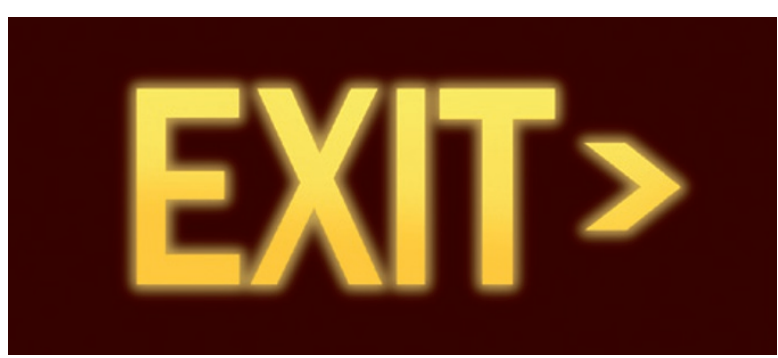

effects on the cell caused by overexpressing fluorescently tagged ESCRT proteins. To overcome this problem, Jouvenet et al. selected cell lines that stably expressed low levels of GFP-tagged ESCRT components that exhibited no adverse cellular effects. Using total internal fluorescence (TIRF) microscopy, the authors observed that the ESCRT-associated protein ALIX (also known as PDCP6IP) was recruited to equine infectious anaemia virus (EIAV) particles that assembled at the plasma membrane at the same time as the viral Gag protein and that ALIX remained associated with the virions throughout the budding process. ESCRT-III components CHMP1B, CHMP4B and CHMP4C as well as VPS4A were only transiently recruited to the HIV-1 and EIAV particles at a late stage, and this usually coincided with the termination of Gag recruitment.

In the second study, Baumgärtel et al. carried out a detailed analysis of the interaction of VPS4A with budding HIV-1 particles. Using TIRF microscopy, they observed that an enhanced-GFP-VPS4A fusion was recruited only transiently at a late stage during HIV-1 budding at the plasma membrane but that it interacted prior to viral release. Cytosolic VPS4A was found to be monomeric, but it formed oligomers with an average of $\sim 30$ units at the budding site, suggesting the recruitment of multiple VPS4A dodecamers.
In the final study, to investigate the specific requirements for ESCRT-III components, Morita et al. used a small interfering RNA approach to systematically deplete cells of each ESCRT-III protein and then monitored the effects on HIV-1 budding. Depletion of any individual CHMP protein had little effect on the efficiency of viral budding, suggesting functional redundancy amongst the CHMP family members. Accordingly, when cells were codepleted of CHMP2A and CHMP2B or co-depleted of CHMP4A, CHMP4B and CHMP4C, there was a substantial reduction in HIV-1 release. Interestingly, co-depletion of CHMP1A and CHMP1B had only minor effects on HIV-1 release, suggesting that despite the transient recruitment of CHMP1B that was observed in the first study, these ESCRT components do not have an essential role in the budding process.

Taken together, these three papers begin to provide a detailed mechanistic understanding of the final steps in the release of enveloped viruses from the host cell.

Andrew Jermy

ORIGINAL RESEARCH PAPERS Jouvenet, N. et al. Dynamics of ESCRT protein recruitment during retroviral assembly. Nature Cell Biol. 13 , 394-401 (2011) | Baumgärtel, V. et al. Live-cell visualization of dynamics of HIV budding site interactions with an ESCRT component. Nature Cell Biol. 13, 469-474 (2011)) | Morita, E. et al. ESCRT-III protein requirements for HIV-1 budding. Cell Host Microbe 9, 235-242 (2011) 